\title{
Communication \\ Material Thickness Classification Using Scattering Parameters, Dielectric Constants, and Machine Learning
}

\author{
Pham-The Hien (10) and Ic-Pyo Hong *(i) \\ Information and Communication Engineering Department, Kongju National University, Cheonan 31080, Korea; \\ pthehien@smail.kongju.ac.kr \\ * Correspondence: iphong@kongju.ac.kr; Tel.: +82-41-521-9199
}

Citation: Hien, P.-T.; Hong, I.-P. Material Thickness Classification Using Scattering Parameters,

Dielectric Constants, and Machine Learning. Appl. Sci. 2021, 11, 10682. https://doi.org/10.3390/ app112210682

Academic Editor:

Pantagiotis G. Asteris

Received: 2 October 2021

Accepted: 8 November 2021

Published: 12 November 2021

Publisher's Note: MDPI stays neutral with regard to jurisdictional claims in published maps and institutional affiliations.

Copyright: (c) 2021 by the authors. Licensee MDPI, Basel, Switzerland. This article is an open access article distributed under the terms and conditions of the Creative Commons Attribution (CC BY) license (https:/ / creativecommons.org/licenses/by/ $4.0 /)$.

\begin{abstract}
Wall-thinning in building structures due to corrosion and surface erosion occurs due to the severe operating conditions and the changing of the surrounding environment, or it can result from poor workmanship and a lack of systematic monitoring during construction. Hence, the continuous monitoring of structures plays an important role in decreasing unexpected accidents. In this paper, a novel method based on the deep neural network and support vector machine approaches is investigated to build up a thickness classification model by incorporating different input features, including the dielectric constants of the material under test, which are extracted from the scattering parameters proceeded by the National Institute of Standards and Technology iterative method. The attained classification results from both machine learning algorithms are then compared and show that both of the models have a good prediction ability. While the deep neural network is the better solution with a large amount of data, the support vector machine is the more appropriate solution when employing small dataset. It can be stated that the proposed method is able to support systematic monitoring as it can help to improve the accuracy of the prediction of material thickness.
\end{abstract}

Keywords: non-destructive testing; thickness classification; support vector machine; deep neural network

\section{Introduction}

According to international norms and regulations, any building materials, products, and elements should have specific physical and strength properties to ensure that the required ultimate and serviceability limit states are met in designed buildings over their whole useful lives [1]. Therefore, there is paramount interest in the constant monitoring of structures for the lifetime assessment of industrial structures [2]. Due to the beneficial characteristics of non-destructive testing (NDT), including the non-destructive nature, repeatability, and economical aspect, it is widely applied in civil engineering, although a disadvantage of this is that the NDT methods are indirect methods. NDT methods can carry out a condition assessment to provide information for the structural performance of the material, including equipment integrity analysis, the corrosion monitoring of structures and equipment, corrosion damage evaluation, fatigue and creep damage prediction, and fitness-for-service evaluations [2].

Several conventional NDT methods are mainly used in routine services to detect the integrity of industrial components [2]. Some methods require expertise and have their limitations. For instance, in the radiography testing method, careful attention needs to be paid to operator safety [3], while eddy current testing only works for conducting materials and requires a highly skillful and experienced operator [2]. On the contrary, the ultrasonic method is widely employed for wall thickness inspections due to its advantages of $[4,5]$ : being nonhazardous and applicable for thickness measurements of thick material, the detection of discontinuity, and the determination of material properties. However, the ultrasonic thickness measurement (UTM) is based on measuring the transit time that is required for a short ultrasonic pulse to travel through the material once, twice, or several 
times, which leads to the need for the UTM system to know the precise physical characteristics, such as the ultrasonic velocity, going through the material [6]. Therefore, often authors investigate and develop new methods for determining ultrasonic velocity $[7,8]$, but usually they present measurement results without the associated measurement uncertainty. Moreover, in [9], the author pointed out that, in ultrasonic inspections, the human factors of time pressure, mental workload, and experience influence the quality of the performance, which can bring about inaccurate inspection results. On the other hand, an electromagnetic (EM) wave has been involved in the thickness inspection of non-metallic materials due to its remarkable features. It does not require a couplant for signal transmission, has a low power consumption, and has a relatively inexpensive price. The material thickness was measured $[10,11]$ by resonant near-field probes whose resonant frequency highly depends on the effective permittivity. This factor will be greatly influenced by the much thicker structure respective to the application discussed herein. Furthermore, it will be very difficult to relate a value (i.e., the resonant frequency) representing the effective permittivity to the liner thickness in the presence of other variables (i.e., wall thickness). In [12,13], a method employing an open-ended waveguide probe radiating into a dielectric structure was proposed for thickness estimation. The slight air gap between the waveguide aperture/flange and the structural wall during measurement may have caused the inaccurate results. Consequently, it leads to the need for reducing the impacts of human factors in measurements as well as the need for skillful inspectors with years of training.

Recently, the machine learning (ML) algorithms and computational tools (GPU acceleration) have been improved significantly and enabled more powerful models that reach the human level in many difficult tasks including image classification and machine translation, and NDT is not an exception. ML systems can be used to automate systems, where a direct algorithmic description is intractable. In [14], four feature selection algorithms were utilized to reduce the sweeping frequencies of the reflected signals before taking them as the dataset to train the surface crack classification model. In [15], two ML models supported measuring the thickness of thin oxide scale using the terahertz time-domain signals as the input feature. In this paper, the material thickness classification models were established by taking the new training dataset extracted from the initial scattering parameters of free space measurement simulations including five input features in the frequency domain: relative permittivity, loss tangent, real and imaginary parts of S21, and frequency. We employed many support vector machine (SVM) kernels and their optimal pair values to construct the SVM model by comparing their results. We also designed and evaluated many deep neural network (DNN) architectures utilizing the same input dataset for the most suitable model. The classification abilities of these SVM and DNN models were then taken into consideration with a conventional method for comparison.

Figure 1a illustrates the traditional method in measuring material thickness by approximating back from the S-parameters and the permittivity. The small dataset and the low accuracy of the approximation process may lead to unstable results. By adopting this proposed method, once the ML models are established with enough dataset, they support a reduction in time spent on a large amount of calculations as well as improve the estimation accuracy, which can bring about stable results. The methodology of this paper is described in Figure 1b. Firstly, the scattering parameters are attained from the simulations before processing through time-domain gating and de-embedding to obtain the exact S-Parameters of the material under test (MUT). The dielectric constants of the MUT are then extracted by the National Institute of Standards and Technology (NIST) iterative method. Thereafter, by incorporating five inputs including frequency, relative permittivity, loss tangent, and real and imaginary parts of S21 into the training phase, the thickness classification models are established. These five inputs extracted from the finite simulated free-space measurements were selected due to their correlations with the material thickness, as illustrated in Section 2. 


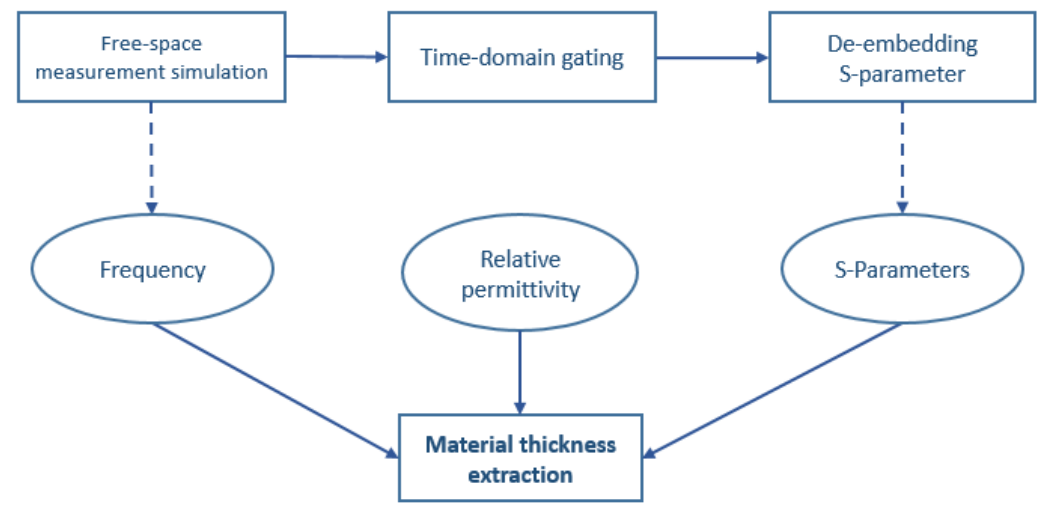

a)

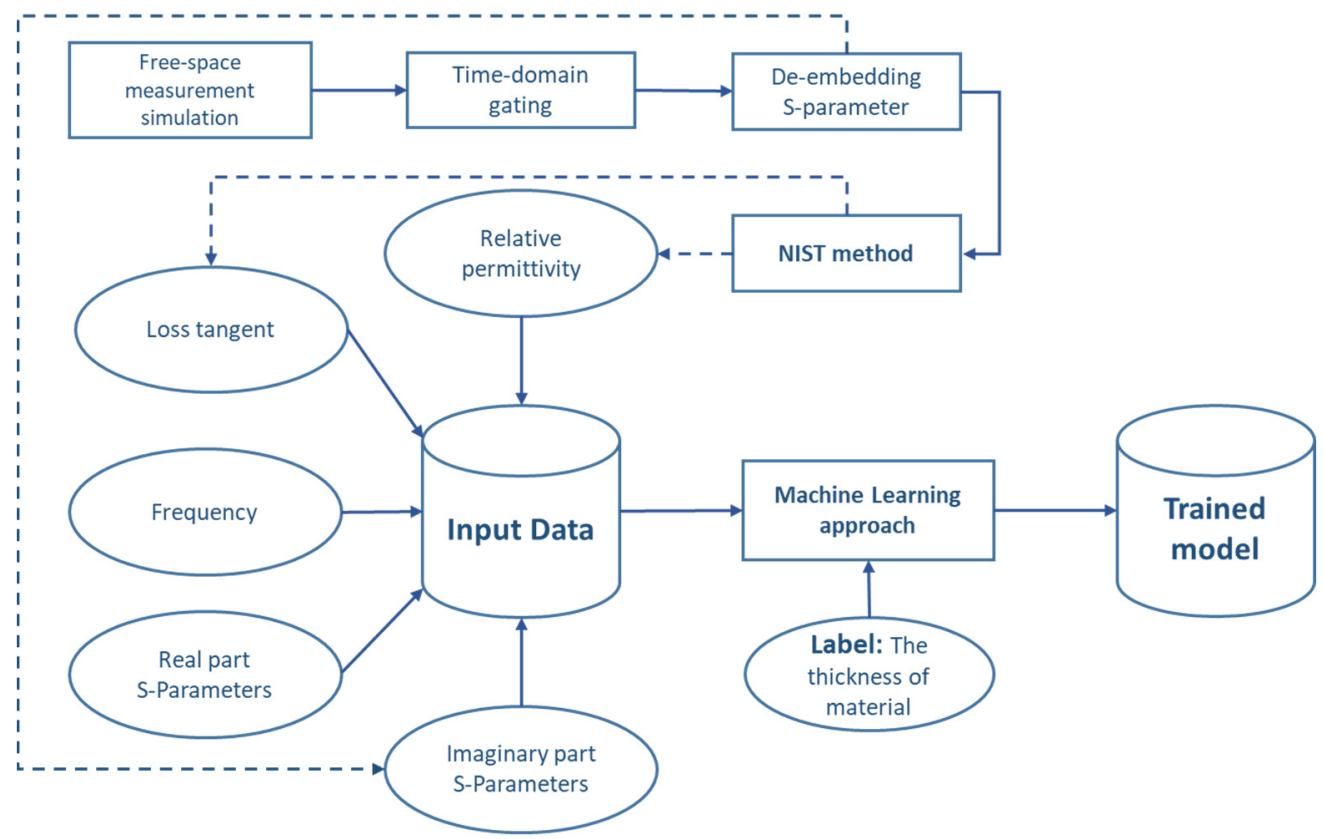

b)

Figure 1. Block diagram of (a) the conventional method and (b) the proposed method.

The paper is organized as follows. After the introduction, Section 2 presents the theory of the dielectric constants extraction algorithm and the method of collecting data for training, the results obtained for numerous sample thicknesses, and the input feature determination. Section 3 details the thickness classification results using the deep neural network (DNN) compared to those which are obtained by the support vector machine (SVM). In Section 4, conclusions are drawn.

\section{Materials and Methods}

\subsection{Dielectric Constant Extraction Method}

The dielectric constants of materials can be obtained by employing the scattering parameters and four conversion techniques: Nicholson-Ross-Weir (NRW), NIST iterative, new non-iterative, and short circuit line [16]. Among these methods, the NRW method is popularly used to derive the dielectric constant of finite thickness material by employing two or all scattering parameters depending on the measurement technique and some mathematical manipulation [17,18]. A challenge encountered when characterizing the electromagnetic properties is the error resulting from the numerical singularity that exists when the sample thickness is equal to an integer multiple of half the wavelength. In our study, we utilize only S21 as the initial data for extracting the relative permittivity and 
loss tangent of low-loss non-magnetic materials as input features of the training model. Therefore, the NIST iterative method seems to be applicable compared to the others since it largely limits the mentioned error of the NRW method and it was proven to be the most conformable method for this type of material [19]. The reflection coefficient, $\Gamma$, can be obtained from

$$
\Gamma=\frac{\frac{\gamma_{0}}{\mu_{0}}-\frac{\gamma}{\mu}}{\frac{\gamma_{0}}{\mu_{0}}+\frac{\gamma}{\mu}}
$$

where the $\gamma_{0}$ is the propagation constant in air, which can be defined as:

$$
\gamma_{0}=j \sqrt{\left(\frac{\omega}{c}\right)^{2}-\left(\frac{2 \pi}{\lambda_{c}}\right)^{2}}
$$

and $\gamma$ is the propagation constant in MUT defined as:

$$
\gamma=j \sqrt{\frac{\omega^{2} \mu_{r} \varepsilon_{r}}{c^{2}}-\left(\frac{2 \pi}{\lambda_{c}}\right)^{2}}=j \sqrt{\varepsilon_{r} \varepsilon_{0} \mu_{0} \omega^{2}-\left(\frac{2 \pi}{\lambda_{c}}\right)^{2}}
$$

where:

- $\mu=\mu_{r} \mu_{0}$ and $\varepsilon=\varepsilon_{r} \varepsilon_{0}$ with $\mu_{r}$ and $\varepsilon_{r}$ are the relative permeability and permittivity, respectively, and $\mu_{0}$ and $\varepsilon_{0}$ are the vacuum permeability and permittivity, respectively;

- $c=1 / \sqrt{\mu_{0} \varepsilon_{0}}$ is the velocity of light;

- $\omega=2 \pi f$ is the angular frequency, with $f$ as the frequency of the signal;

- $\lambda_{c}$ is the cutoff wavelength.

Since the MUTs are non-magnetic materials, $\mu_{r}=1$. Then, the reflection coefficient in (1) can be rewritten as:

$$
\Gamma=\frac{\gamma_{0}-\gamma}{\gamma_{0}+\gamma}
$$

The transmission coefficient is:

$$
T=e^{-\gamma d}=e^{\left(-j d \sqrt{\varepsilon_{r} \varepsilon_{0} \mu_{0} \omega^{2}-\left(\frac{2 \pi}{\lambda_{c}}\right)^{2}}\right)}
$$

with $d$ as the MUT thickness.

By solving Equations (4) and (5), the relative permittivity can be determined as:

$$
F\left(\varepsilon_{r}\right)=\frac{S_{21}+S_{12}}{2\left(1-T^{2} \Gamma^{2}\right)}-T\left(1-\Gamma^{2}\right) e^{-j \gamma_{0}\left(L_{a i r}-d\right)}
$$

The relative complex dielectric permittivity can then be computed from the scattering parameters by solving Equation (6) using the Newton-Raphson iterative approach $[19,20]$. The algorithm is considered converged when $F\left(\varepsilon_{r}\right)=0$.

\subsection{Multilayer Structure Simulation}

To collect adequate data for constructing the classification model, a multilayer structure simulation method is investigated in this work which is equivalent to the free space measurement. The structure includes three layers of air and MUT with the incidence angle $\theta$ as $0^{\circ}$, as illustrated in Figure 2. 


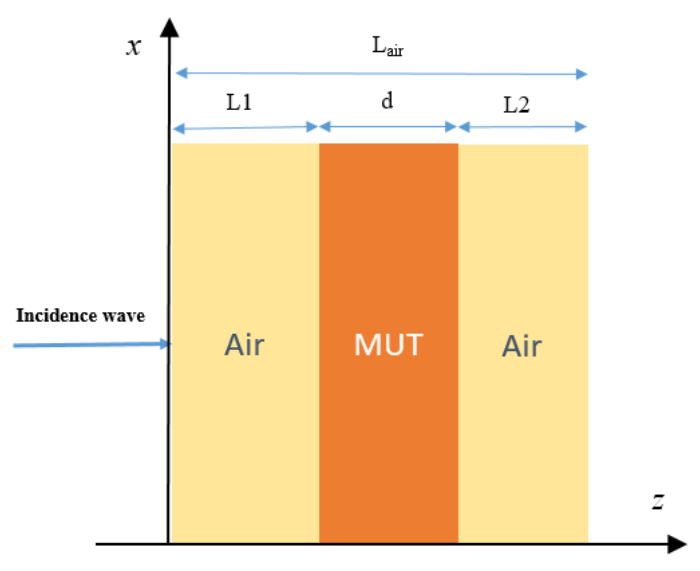

Figure 2. Multilayer structure of simulated measurement.

According to the multilayer structure above, the $A B C D$ matrix of the equivalent circuit for the two-port network is obtained for the whole structure by the following equations [21]:

$$
\left[\begin{array}{cc}
A & B \\
C & D
\end{array}\right]=\left[\begin{array}{ll}
1 & 0 \\
0 & 1
\end{array}\right]\left[\begin{array}{cc}
\cos \left(k_{z n} d\right) & \frac{j \sin \left(k_{z n} d\right)}{Y_{d}^{\{T E, T M\}}} \\
j Y_{d}^{\{T E, T M\}} \sin \left(k_{z n} d\right) & \cos \left(k_{z n} d\right)
\end{array}\right]\left[\begin{array}{ll}
1 & 0 \\
0 & 1
\end{array}\right]
$$

where:

- $k_{z n}=\sqrt{\varepsilon_{r} k_{0}^{2}-k_{0}^{2} \sin ^{2} \theta}$ is the wavenumber of MUT layer with $k_{0}=\beta=2 \pi / \lambda$ as the wavenumber of the first air layer, $\theta$ as the incidence angle of the incoming signal, and $\varepsilon_{r}$ as the relative permittivity of MUT;

- $\quad Y_{d}^{T E}=k_{z n} / \omega \mu_{r} \mu_{0}$ and $Y_{d}^{T M}=\omega \varepsilon_{r} \varepsilon_{0} / k_{z n}$ are the admittances in TE and TM polarizations, respectively. Since the incidence angle $\theta$ is $0^{\circ}$, either $Y_{d}^{T E}$ or $Y_{d}^{T M}$ can be chosen for the calculation;

- $\quad d$ is the thickness of MUT.

From the $\mathrm{ABCD}$ matrix, the scattering parameters can be deduced by the equivalent circuits for the two-port network [21] as follows:

$$
\begin{aligned}
& S_{11}=\frac{A+B Y_{0}-C / Y_{0}-D}{A+B Y_{0}+C / Y_{0}+D} \\
& S_{21}=\frac{2}{A+B Y_{0}+C / Y_{0}+D}
\end{aligned}
$$

where $Y_{0}=k_{z n} / \omega \mu_{0}=\omega \varepsilon_{0} / k_{z n}$ (as the incidence angle $\theta$ is $0^{\circ}$ ).

Eventually, by adopting the appropriate relative permittivity, relative permeability, dielectric dissipation, magnetic dissipation, and the thickness of an MUT, one can extract the simulated scattering parameters data of the free-space measurement based on the above procedure.

\subsection{Data Accumulation and the Process of the Proposed Method}

By following the aforementioned method, 80 simulated multilayer structures were created. In each simulated structure, $101 \mathrm{X}$-Band frequency points in the range of $7 \mathrm{GHz}$ to $13 \mathrm{GHz}$ are taken into account as a signal goes through the structure. The MUTs are considered as the low-loss dielectric materials whose relative permeability is around 1 and relative permittivity values are distinct and close to five base values: 2, 2.5, 3, 3.5, and 4 . They are closely approximated to the base value. For example, if the base value is 2, the distinct actual value is randomly chosen in the range of 1.9 to 2.1. The MUT thicknesses variating from $7 \mathrm{~mm}$ to $10.5 \mathrm{~mm}$ with a small increasing step of $0.5 \mathrm{~mm}$ result in eight values: 7, 7.5, 8, 8.5, 9, 9.5, 10, and $10.5 \mathrm{~mm}$. Each thickness was combined with ten 
distinct permittivity values extracted by the above random method approximating from all five base values (two permittivities were approximated from each base value for each thickness). As a result, 80 combinations of all thicknesses and permittivities were involved in the simulation. The design of the multilayer structure is shown in Table 1.

Table 1. Input parameters of simulation procedure.

\begin{tabular}{cc}
\hline Parameters & Values \\
\hline Frequency range & $7-13 \mathrm{GHz}$ \\
Relative permittivity & $2-4$ \\
Dielectric dissipation & 0.01 \\
Relative permeability & $1-1.02$ \\
Magnetic dissipation & 0.0001 \\
Thickness of MUT & $7-10.5 \mathrm{~mm}$ \\
Distance from antenna to MUT & $5 \mathrm{~cm}$ \\
\hline
\end{tabular}

The simulated geometry has been mathematically modeled as three stratified regions, each with differing EM propagation parameters. Figure 2 illustrates that the interested MUT volume is surrounded by two air layers where the extracted scattering parameters of each structure contain both air layers and MUT volume. In order to obtain the exact scattering parameters of the MUT for the further steps, the de-embedding procedure must be implemented $[22,23]$. The process of de-embedding the MUT fixture using Tparameters [24] is as follows:

$$
\begin{aligned}
& T_{\text {Measured }}=T_{\text {air } 1} T_{\text {MUT }} T_{\text {air } 2} \\
& T_{\text {MUT }}=T_{\text {air } 1}^{-1} T_{\text {Measured }} T_{\text {air } 2}^{-1}
\end{aligned}
$$

where:

- $\quad T_{\text {Measured }}$ is converted from the scattering parameter of the whole structure;

- $T_{a i r}$ is calculated by converting the scattering matrix defined in (12) for an idealized section of air of length $L$ ( $L$ is the distance from antenna to MUT).

$$
S_{a i r}=\left[\begin{array}{cc}
0 & e^{-j 2 \pi f L} \\
e^{-j 2 \pi f L} & 0
\end{array}\right]
$$

In this study, we conducted a real free space measurement and compared its results with those of the simulation to validate the difference of the de-embedding and NIST iterative methods on both types of data. The free space measurement is depicted in Figure 3, where the bistatic setup is used to measure an acrylic specimen whose permittivity is around 2.5 and thickness is $10 \mathrm{~mm}$. The $\mathrm{X}$-band signal with a frequency range of $8-12.4 \mathrm{GHz}$ is caused by the MUT.

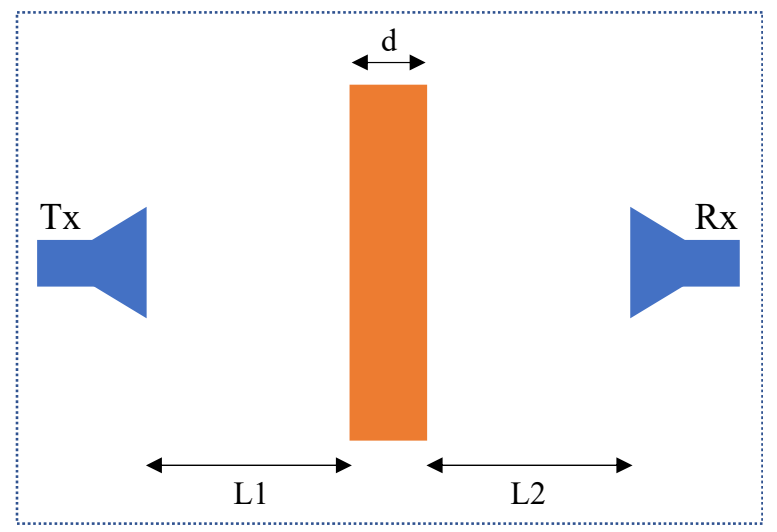

Figure 3. The bistatic experiment setup. 
Figure 4 below shows the S11 and S21 of one multilayer structure after performing the de-embedding process, which are now ready to be used in the NIST iterative method for the dielectric constants of the MUT. Figure 5 illustrates the dielectric constants of the MUT by applying the NIST iterative method to the de-embedded scattering parameters from the data of the simulation and the real measurement. It can be seen that the dielectric constants attained from simulated and measured data are closed to each other and to the actual values of the MUT. The NIST iterative method provides accurate outputs as well as helps to prevent the inconsistent results caused by the significant deterioration of S11 if using the NRW method [17].

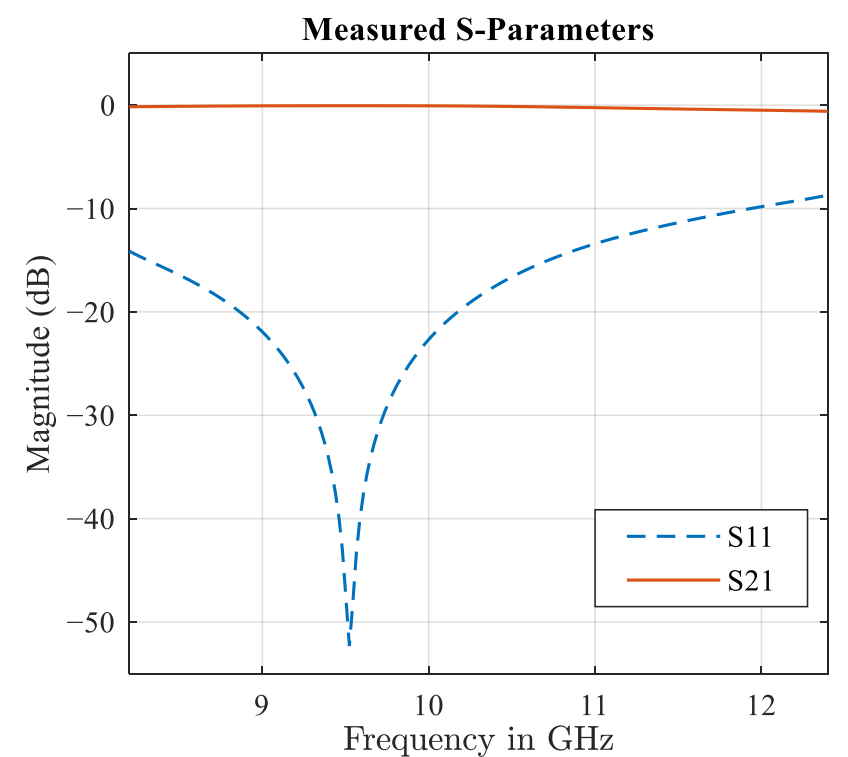

(a)

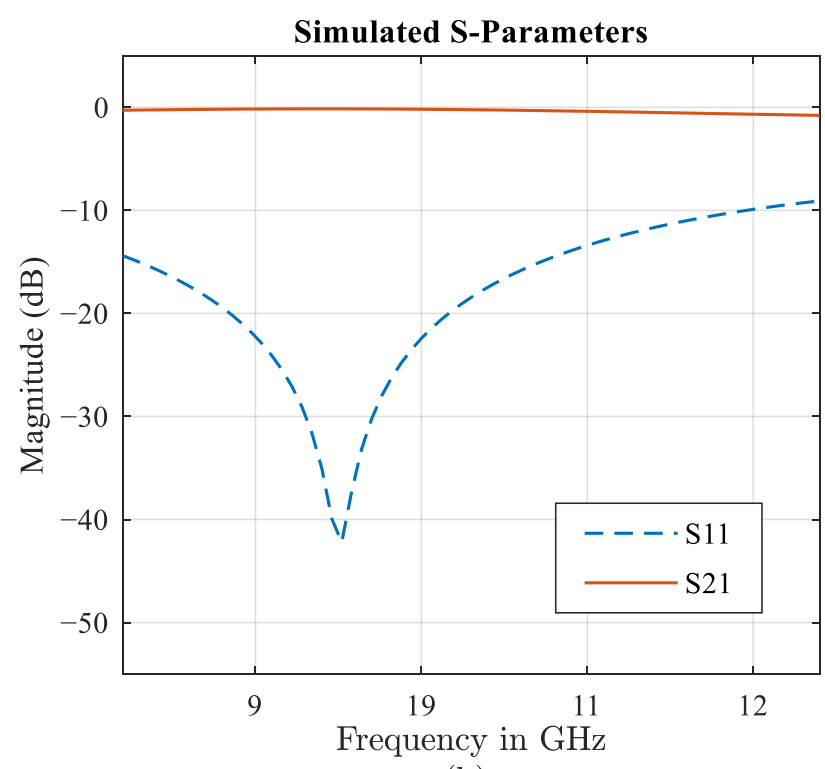

(b)

Figure 4. The de-embedded S11 and S21 of MUT from (a) measurement and (b) simulation.

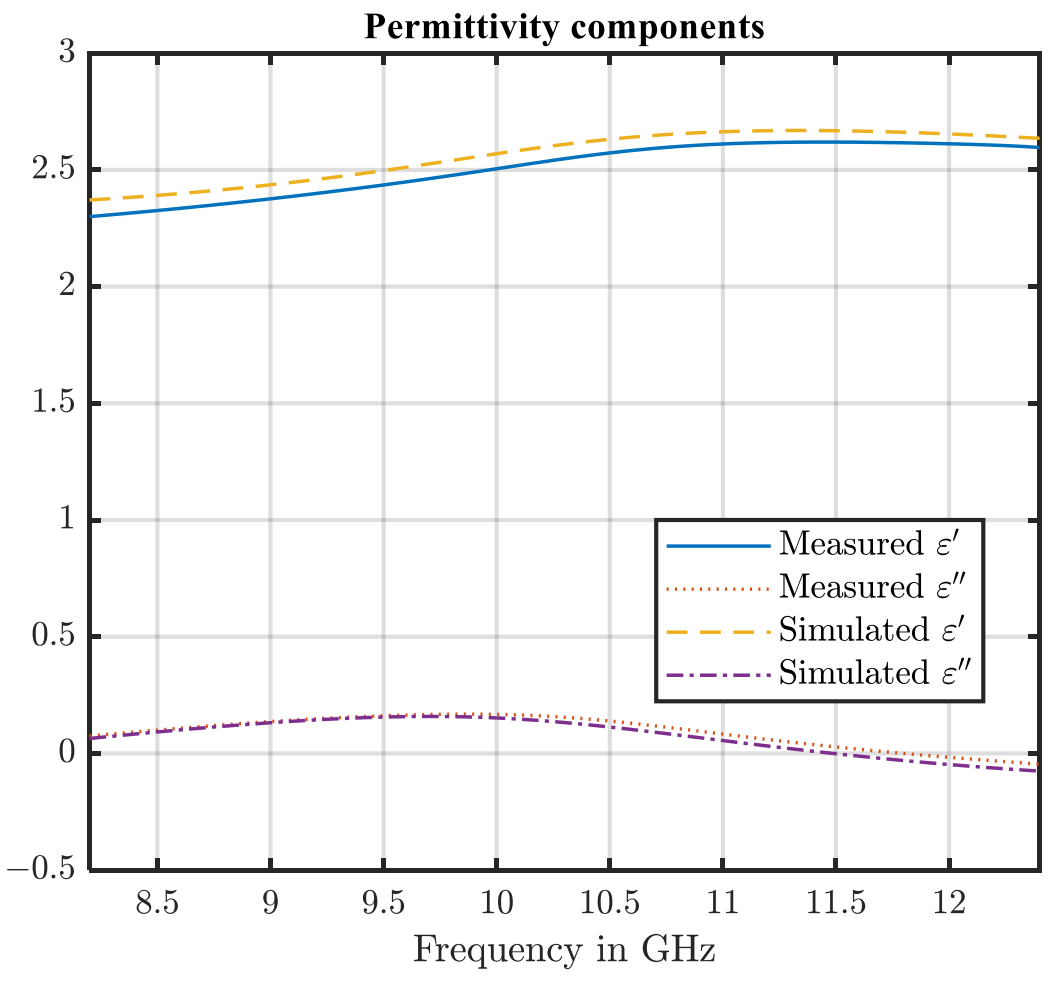

Figure 5. Dielectric constant of MUT obtained by the NIST iterative method. 
The results of the methods are slightly similar, which can confirm that the simulation structure in [21] is suitable for our research. The small differences may occur due to the equipment errors, but this is negligible. Due to the requirement of an adequate dataset for training the models, the simulations were conducted for various thicknesses and types of material characteristics. One can notice that there are non-linear correlations between the scattering parameter, dielectric constants, and the MUT thickness through the deembedding process and the NIST iterative method. A small change in the thickness of the simulated MUT can cause the different results extracted from these methods. Therefore, the ML can help to improve the estimation accuracy by overcoming the mathematical approximation when employing an adequate dataset for training. This suggests that the frequency, scattering parameters, and dielectric constants can be used to classify the specimen thickness. Thus, in this work, we extracted five inputs to put into the ML training model: frequency, relative permittivity, loss tangent, and real and imaginary parts of S21.

\section{Classification with Machine Learning and Results}

\subsection{Machine Learning Classification Models}

Neural networks (NNs) are the mathematical models used to simulate the biological neurons and to perform parallel information processing in a simple and objective way. Single hidden-layer feed-forward NNs typically contain one input layer, a hidden layer and an output layer, while a DNN has more than one hidden layer.

In this study, we built up an eight-layered DNN with six hidden layers. The input layer has five nodes that are the same number as the types of selected input features. For hidden layers, 100 nodes are located in each one while the output layer contains eight nodes since the data should be classified into eight thickness classes. This model was constructed after comparing different designs. Generally, the accurate classification rate is proportional to the number of hidden layers and the number of nodes in each layer, but it also leads to the remarkable increase in training duration if the architecture of the model is too complex. Therefore, after many considerations, the proposed design in this research is reasonable for this classification problem since it offers good classification results and the proper training time.

The DNN with a large number of hidden layers and millions of learnable parameters usually suffer from over-fitting without regulation. There are several techniques to overcome this issue including early stopping, $l_{1}$, and $l_{2}$ regulation and max norm, etc., but drop-out is the most popular one [25]. In this DNN model, one drop-out layer was implemented on each of the fully connected layers before the output layer. This helps to avoid overfitting by randomly dropping units from the network during training [25-27] as it can prevent co-adaptation between layers and nodes of deep feedforward neural networks in each training case. The nodes to drop are chosen randomly in this study with a probability of 0.5 [25]. The activation function in the hidden layers is rectified linear unit $(\operatorname{ReLU})$ that is represented as $\operatorname{ReLU}(\mathrm{x})=\max (0, \mathrm{x})$ since its performance is already proposed for DNN $[26,28,29]$. The soft-max function is used as the activation function in the output layer which is suitable for our multilabel classification problem. The solver stochastic gradient descent with momentum was chosen after the optimization process along with the learning rate of $10^{-3}$ and a mini-batch size of 500 . The structure of our DNN model is shown in Figure 6. 


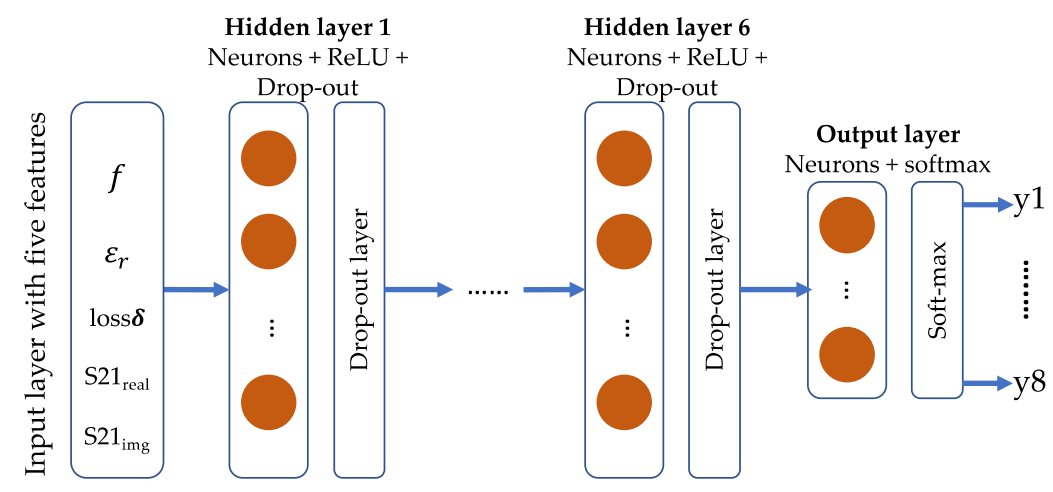

Figure 6. The overall DNN structure. The hidden layers comprise multiple fully connected, ReLU and Drop-out layers. The output layer comprises the fully connected layer followed by a soft-max.

The SVM algorithms can solve classification and regression problems. This paper also considers the study of SVM to classify eight thicknesses by employing the attained dataset as described in Section 2.3. The software used in this work is MATLAB with the LIBSVM library. Four well-known kernels, linear, polynomial, sigmoid, and radial basis function (RBF), were used to train the models due to their great impacts on the performance of SVM. Each kernel can be fitted to the corresponding model by adjusting the corresponding parameters: $\operatorname{cost}(\mathrm{C})$ and gamma $(\gamma)$. These parameters were optimized using the grid search method in order to maximize the accuracy rates of the thickness classification [30,31]. To avoid overfitting, a 10-fold cross validation was implemented. The classification accuracy is calculated as:

$$
\operatorname{Accuracy}(T)=\frac{\sum_{i=1}^{T} A \operatorname{ssess}\left(t_{i}\right)}{|T|} \times 100 \%, t_{i} \in T
$$

where $T$ is the set of test samples and

$$
\operatorname{Assess}\left(t_{i}\right)=\left\{\begin{array}{c}
1 \text { if } t_{i} \text { is correctly classified } \\
0 \text { if } t_{i} \text { is incorrectly classified }
\end{array}\right.
$$

\subsection{Results}

The extracted data from the simulations have been exploited to conclude whether the proposed classification models can detect the variation of the MUT thickness. The procedure started with the training classification models with eight classes of all thicknesses of simulated specimens. The models obtained after the training were then tested to verify their accuracy.

The 80 simulated structures with 101 frequency points in each one resulted in 8080 data samples in which each sample consists of five of the aforementioned input features and the corresponding thicknesses. The dataset was then split into three sets by selecting $70 \%$ of the total acquisitions for the training phase, $20 \%$ to validate the trained model, and the distinct remaining $10 \%$ for the testing set. The validation was conducted by putting $20 \%$ of the acquisitions to the trained models and comparing the attained classifications with the corresponding simulated MUT thicknesses to frequently evaluate the model. The distinct testing set was then taken into account for the final evaluation after the models had been completely trained and validated. The same amount of data in the training set, validation set, and testing set were used in the training phase, validation, and testing phase for both DNN and SVM classification models in this paper.

The flowchart of the investigational platform is shown in Figure 7. First, the scattering parameters and dielectric constants of MUTs were acquired as described in Section 2. Subsequently, two predictive models were trained using the input features from the previous procedure. The evaluation of training and prediction results were then used to compare the models. 


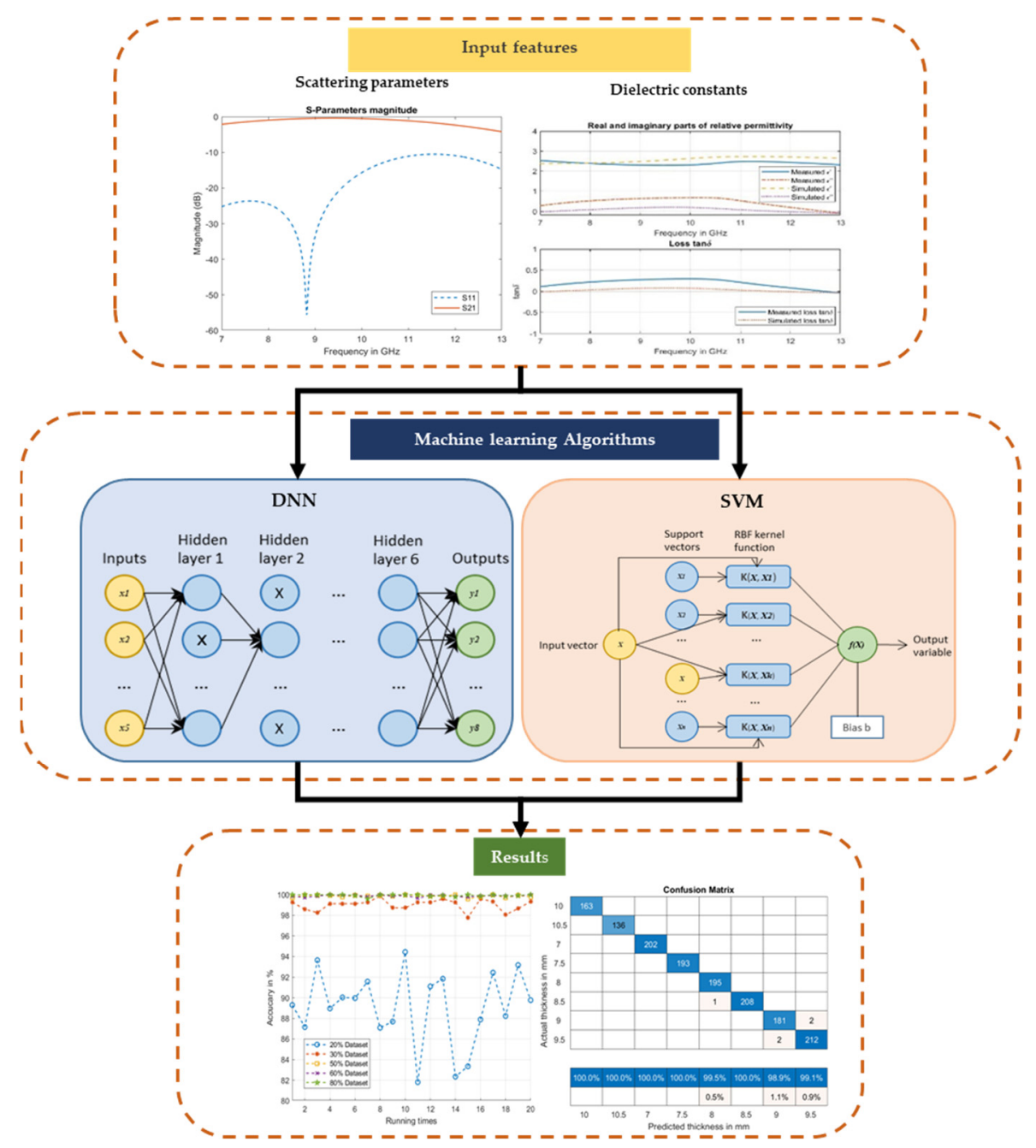

Figure 7. The investigational platform.

In terms of the aforementioned SVM training above and based on the 10-fold cross validation results, the grid search was able to find the optimal pair value of both parameters ( $\mathrm{C}$ and $\gamma$ ) of four kernels. The overall summary of various kernels is listed in Table 2. It can clearly be seen that the best SVM model values using the RBF kernel with the optimal $\mathrm{C}$ and $\gamma$ after grid search are 224.81 and 0.26986 , respectively. The reason for this is that SVM adopting RBF kernel had the highest accuracy compared to its competitors while the linear kernel provided the lowest classification ability. However, it should be noted that the search interval is also a problem. Due to the large search region, it will waste many computational resources. For example, the polynomial kernel required a relatively longer computational time than the others when employing $70 \%$ of datasets with a duration of around $3 \mathrm{~h}$ while the other kernels needed approximately $2 \mathrm{~h}$ to finish searching. However, too small a search region may lead to an unsatisfactory outcome. Therefore, one must correctly analyze the search region before conducting the optimization simulations.

Table 2. Optimal pair value of four SVM kernels.

\begin{tabular}{ccccc}
\hline \multirow{2}{*}{ Kernel Functions } & \multicolumn{2}{c}{ Optimal Pair Value } & \multirow{2}{*}{ Accuracy (\%) } & 10-Fold Loss \\
\cline { 2 - 3 } & $\boldsymbol{C}$ & $\gamma$ & & \\
\hline Linear & 1.7079 & 0.03335 & 81.3 & 0.18907 \\
Polynomial & 0.20641 & 0.39028 & 99.1 & 0.0016732 \\
Sigmoid & 181.019 & 0.31079 & 98.6 & 0.0019681 \\
RBF & 224.81 & 0.26986 & 99.7 & 0.0011154 \\
\hline
\end{tabular}


The results of the SVM classification when adopting the RBF kernel are illustrated in Figure 8a with different percentages of data being trained and tested. For each data partition, the algorithm was independently executed 20 times using the training/testing data, which were randomly selected for each time. The improvement in the accuracy corresponds to the increased training dataset in the results, which is acceptable with our expectations. The accuracy of around $90 \%$ can be inferred by using our smallest training set of 1500 data samples, only one-fifth of the total inputs. The small variations in all results point to the robustness of the SVM algorithm.

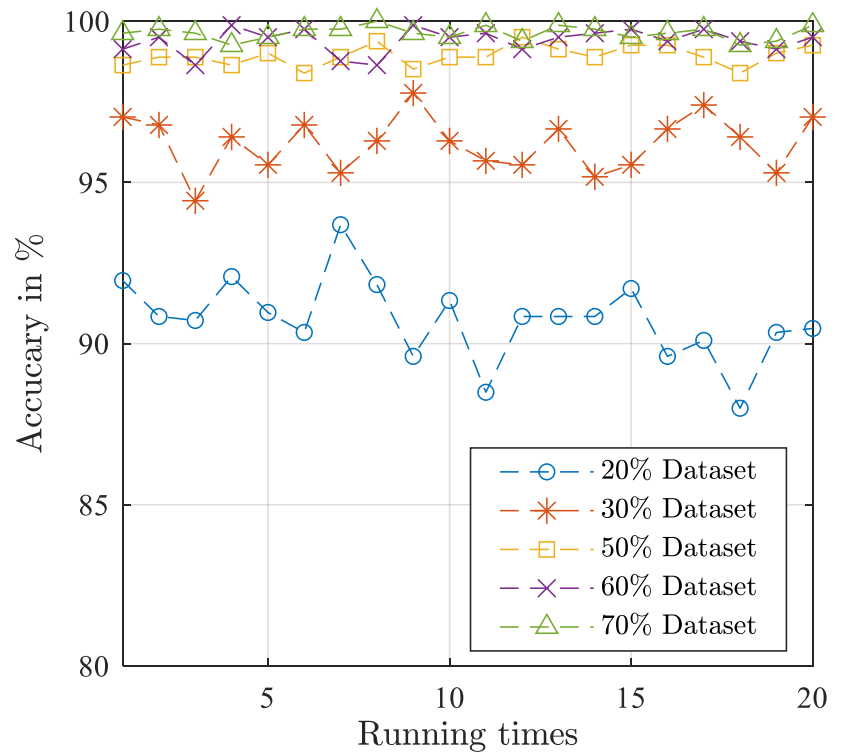

(a)

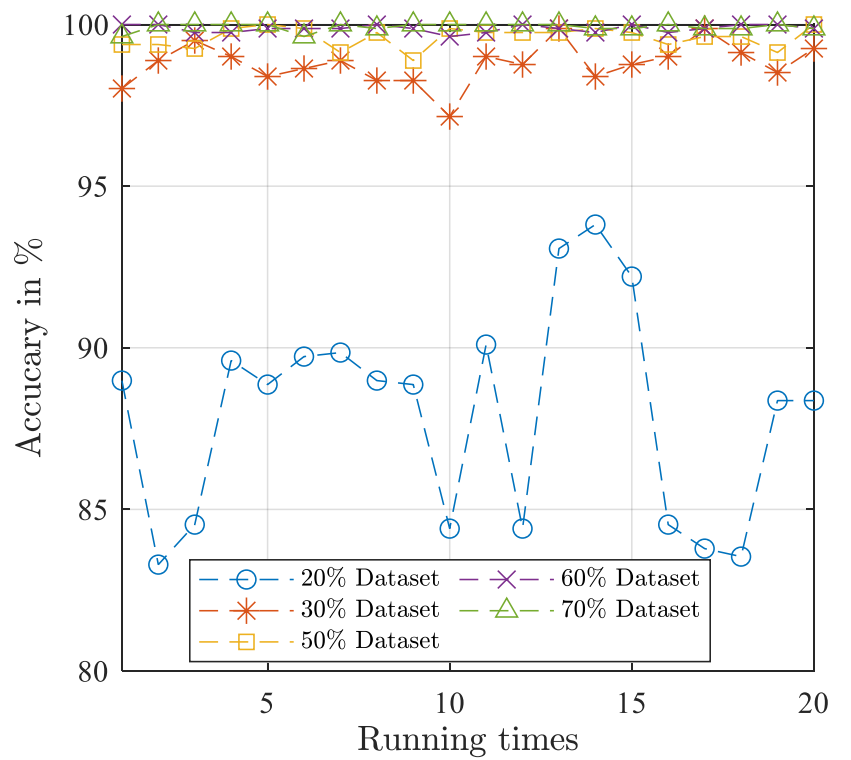

(b)

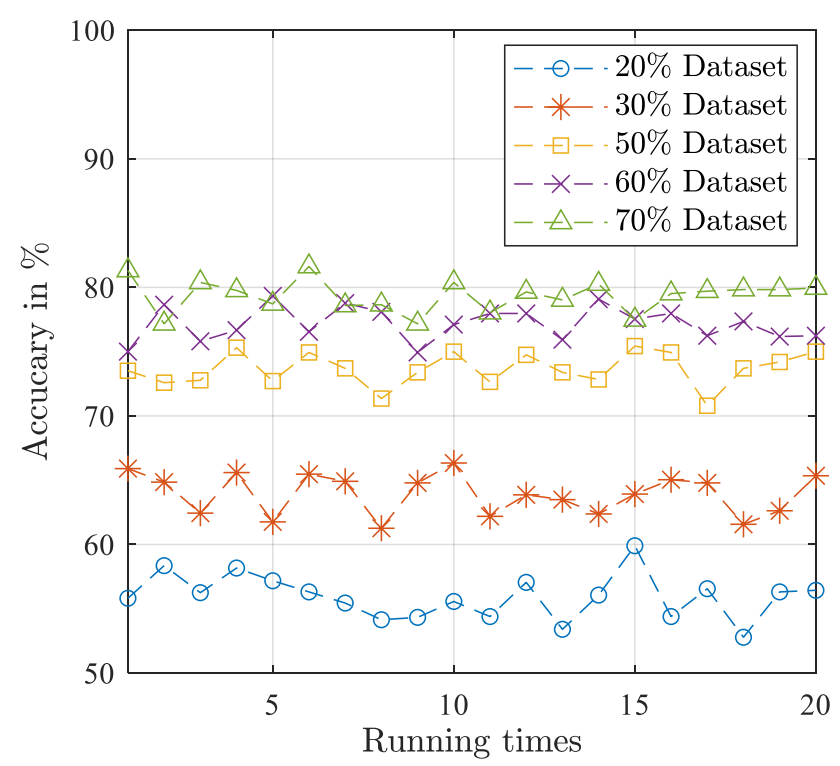

(c)

Figure 8. The classification accuracy versus the number of running times: (a) SVM algorithm, (b) DNN algorithm, and (c) decision tree algorithm.

The DNN model was also executed 20 times independently for each dataset comparison as in Figure 8b. It can clearly be seen that the performance of SVM using the lowest amount of data is higher and more stable than that of the DNN model. In contrast, the DNN utilizes a high percentage of datasets better as most of the accuracies are over 98\%. Moreover, Figure 8c demonstrates that both the proposed models have significantly 
higher abilities than the decision tree algorithm, which represents a linear classification at all percentages of the dataset which means that both of these classification models are reasonable choices for the non-linear problem of this study.

Figure 9 shows two testing results of each classification model, with $20 \%$ and $70 \%$ datasets on a logarithmic scale including the correct and incorrect prediction rates. All of the incorrect predictions classify the thicknesses into the wrong classes, which are adjacent to the actual thicknesses. Fewer misclassifications from the SVM model with the $20 \%$ dataset can be observed due to the low incorrect prediction rate in Figure 9a compared to that of the DNN in Figure 9b. In contrast, the DNN obtains the higher correct prediction rate when employing a 70\% dataset as in 9d, although the SVM model also accomplishes good results with only two thicknesses having incorrect predictions, as in Figure 9c.

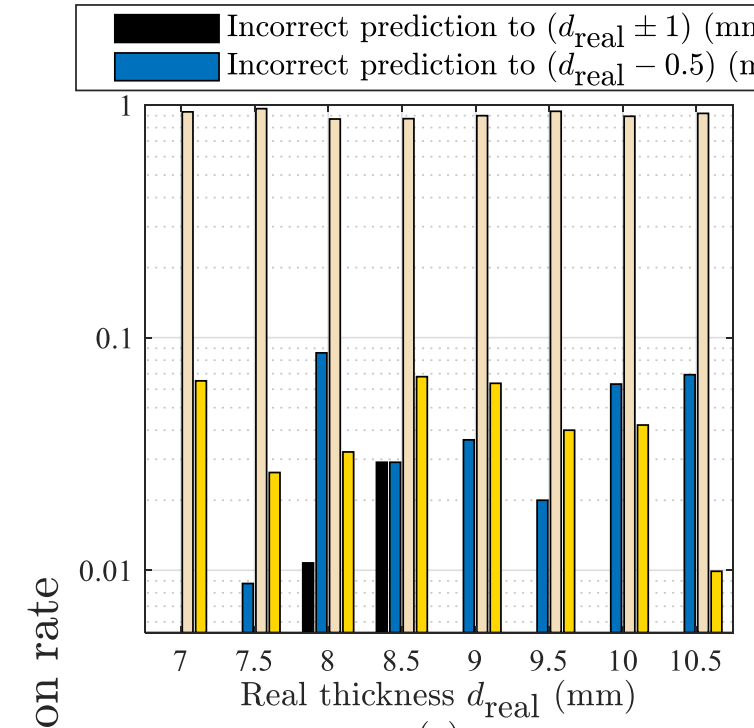

(a)

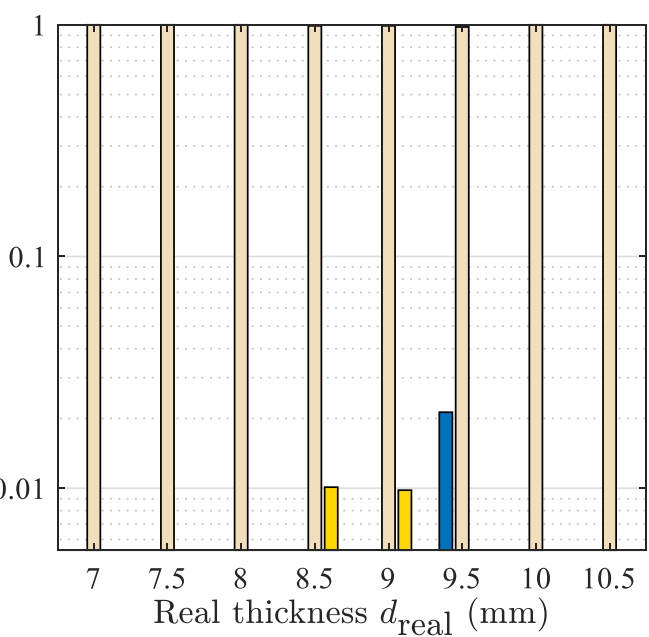

(c)

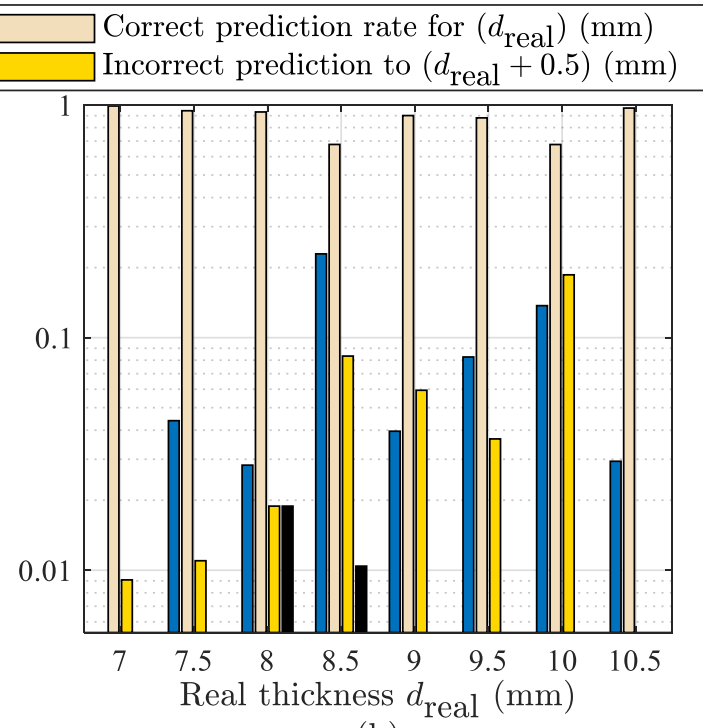

(b)

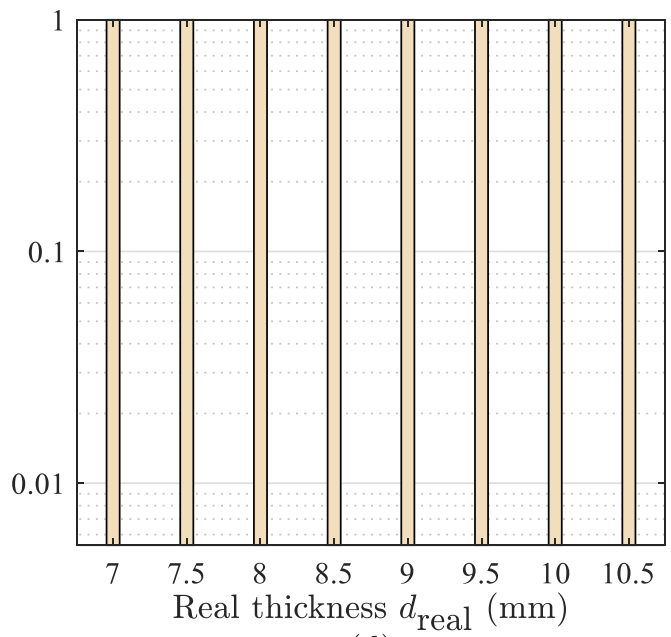

(d)

Figure 9. The classification testing results of (a) SVM with $20 \%$ dataset (b) DNN with $20 \%$ dataset (c) SVM with $70 \%$ dataset and (d) DNN with $70 \%$ dataset.

Finally, we inferred the material thickness by relating back to the conventional method as in Figure 1a for comparison. The NIST iterative method employed the same dataset for extracting the thickness from each set of input features. The obtained thicknesses were evaluated with the actual ones to determine the average accuracy of each thickness. These results were then compared to those attained from two proposed ML models whose 
training phases utilized $70 \%$ of the dataset. Table 3 reveals that two trained models work more effectively than the traditional approach as they provide a higher accuracy rate for all thicknesses.

Table 3. Thickness estimation accuracy among the NIST iterative method, the SVM model, and the DNN model.

\begin{tabular}{cccc}
\hline \multirow{2}{*}{$\begin{array}{c}\text { Thickness } \\
(\mathbf{m m})\end{array}$} & \multicolumn{3}{c}{ Average Estimation Accuracy (\%) } \\
\cline { 2 - 4 } & NIST Iterative & SVM & DNN \\
\hline 7 & $98.5 \%$ & $100 \%$ & $100 \%$ \\
7.5 & $98.5 \%$ & $100 \%$ & $100 \%$ \\
8 & $98.2 \%$ & $100 \%$ & $100 \%$ \\
8.5 & $96.7 \%$ & $99.9 \%$ & $100 \%$ \\
9 & $97.2 \%$ & $99.1 \%$ & $99.5 \%$ \\
9.5 & $96.5 \%$ & $97.8 \%$ & $100 \%$ \\
10 & $96.2 \%$ & $100 \%$ & $100 \%$ \\
10.5 & $97.2 \%$ & $100 \%$ & $100 \%$ \\
\hline
\end{tabular}

\section{Conclusions}

In the present article, we introduce a comparative study of two ML algorithms in material thickness classification using five input features, including the extracted dielectric constants from the NIST iterative method in order to improve the prediction of material thickness. Based on the initial scattering parameters, the new five features in the frequency domain can be integrated into two ML algorithms to train the classification model for detecting the thicknesses of test specimens. Four SVM kernels and their optimal pair values were determined by using the grid search method to build up the SVM model. The eight-layered DNN model was established and compared to the SVM one using the same dataset. While the SVM algorithm leads to a higher performance by employing a small amount of data, the DNN algorithm achieves a better performance with a large quantity of data. We also compared the results between the proposed method and the conventional approach. The comparison results state that once the classification model is constructed, it can help to decrease the calculation time as well as enhance the accurate and stable estimations. The high accuracy rate of classification results can show that the selected features are slightly representative and it strongly confirms that the proposed method can be useful in predicting the unknown thickness of a material, which is possibly integrated into the electromagnetic NDT methods.

In future work, the monostatic measurement setup and regression model will be included instead of the bistatic setup and classification for an estimate of the thickness of specimens. Furthermore, the copula theory techniques will be studied to capture the non-linear dependency between the aforementioned correlated input features and an input feature selection method, such as principle component analysis (PCA), will be adopted to optimize this classification problem.

Author Contributions: Experimental work: P.-T.H.; conceptualization: P.-T.H. and I.-P.H.; data processing and statistical analysis: P.-T.H.; writing —original draft preparation: P.-T.H.; review of the text: I.-P.H.; writing—review and editing: I.-P.H.; supervision: I.-P.H.; project administration: I.-P.H.; funding acquisition: I.-P.H., All authors have read and agreed to the published version of the manuscript.

Funding: This research was funded in part by the Basic Science Research Program under Grant 2020R1I1A3057142, and in part by the Priority Research Centers Program through the National Research Foundation of Korea under Grant 2019R1A6A1A03032988.

Institutional Review Board Statement: Not applicable.

Informed Consent Statement: Not applicable.

Data Availability Statement: Not applicable. 
Conflicts of Interest: The authors declare no conflict of interest.

\section{References}

1. Runkiewicz, L. Application of non-destructive testing methods to assess properties of construction materials in building diagnostics. Archit. Civ. Eng. Environ. 2009, 2, 79-86.

2. Einav, I. Non-Destructive Testing for Plant Life Assessment; International Atomic Energy Agency: Vienna, Austria, $2005 ;$ pp. 1-61.

3. International Atomic Energy Agency. Development of Protocols for Corrosion and Deposits Evaluation in Pipes by Radiography; IAEA-TECDOC-1445; International Atomic Energy Agency: Vienna, Austria, 2006.

4. Haibo, L.; Yongqing, W.; Meng, L.; Tongyu, Z.; Baoliang, L. Thickness Measurement Using Ultrasonic Scanning Method for Large Aerospace Thin-Walled Parts. In Proceedings of the 2019 IEEE 5th International Workshop on Metrology for AeroSpace (MetroAeroSpace), Turin, Italy, 19-21 June 2019; pp. 243-247. [CrossRef]

5. Zhongzhu, L.; Chunguang, X.; Bolong, M. Application of SAGE Algorithm to Estimate Thin Layer Material's Thickness in Ultrasonic NDE. In Proceedings of the 2011 International Conference on Electronic \& Mechanical Engineering and Information Technology, Harbin, China, 12-14 August 2011; pp. 2869-2872. [CrossRef]

6. Bustillo, J.; Achdjian, H.; Arciniegas, A.; Blanc, L. Simultaneous determination of wave velocity and thickness on overlapped signals using Forward Backward algorithm. NDT E Int. 2017, 86, 100-105. [CrossRef]

7. Loosvelt, M.; Lasaygues, P. A wavelet-based processing method for simultaneously determining ultrasonic velocity and material thickness. Ultrasonics 2011, 51, 325-339. [CrossRef] [PubMed]

8. Kuhnicke, E.; Wolf, M.; Kummritz, S. Approach for simultaneous determination of thickness and sound velocity in layered structures based on sound field simulations. AIP Conf. Proc. 2017, 1806, 1-10. [CrossRef]

9. Bertovic, M.; Gaal, M.; Müller, C.; Fahlbruch, B. Investigating human factors in manual ultrasonic testing: Testing the human factors model. Insight-Non-Destr. Test. Cond. Monit. 2011, 53, 673-676. [CrossRef]

10. Lee, C.-S.; Yang, C.-L. Thickness and permittivity measurement in multi-layered dielectric structures using complementary split-ring resonators. IEEE Sens. J. 2014, 14, 695-700. [CrossRef]

11. Li, Y.; Bowler, N.; Johnson, D.B. A resonant microwave patch sensor for detection of layer thickness or permittivity variations in multilayered dielectric structures. IEEE Sens. J. 2011, 11, 5-15. [CrossRef]

12. Kempin, M.; Ghasr, M.T.; Case, J.T.; Zoughi, R. Modified waveguide flange for evaluation of stratified composites. IEEE Trans. Instrum. Meas. 2014, 63, 1524-1534. [CrossRef]

13. Ghasr, M.T.; Horst, M.J.; Lechuga, M.; Rapoza, R.; Renoud, C.J.; Zoughi, R. Accurate one-sided microwave thickness evaluation of lined-fiberglass composites. IEEE Trans. Instrum. Meas. 2015, 64, 2802-2812. [CrossRef]

14. Moomen, A.; Ali, A.; Ramahi, O.M. Reducing sweeping frequencies in microwave ndt employing machine learning feature selection. Sensors 2016, 16, 559. [CrossRef] [PubMed]

15. Xu, Z.; Ye, D.; Chen, J.; Zhou, H. Novel terahertz nondestructive method for measuring the thickness of thin oxide scale using different hybrid machine learning models. Coatings 2020, 10, 805. [CrossRef]

16. Kerim, A.A. Novel Technique for Measuring the Complex Dielectric Constant and Permeability. In Proceedings of the 2016 17th International Symposium on Antenna Technology and Applied Electromagnetics (ANTEM), Montreal, QC, Canada, 10-13 July 2016; pp. 1-3. [CrossRef]

17. Alexandre, N.V.; Gustavo, M.D.; Cynthia, J. The Step by Step Development of NRW Method. In Proceedings of the 2011 SBMO/IEEE MTT-S International Microwave and Optoelectronics Conference (IMOC 2011), Natal, Brazil, 29 October-1 November 2011; pp. 738-742. [CrossRef]

18. Olli, L.; Stanislav, I.M.; Sergei, A.T. A Stepwise Nicolson-Ross-Weir-based material parameter extraction method. IEEE Antennas Wirel. Propag. Lett. 2011, 10, 1295-1298. [CrossRef]

19. Piuzzi, E.; Cannazza, G.; Cataldo, A.; Chicarella, S.; De Benedetto, E.; Frezza, F.; Pisa, S.; Prontera, S.; Timpani, F. Measurement system for evaluating dielectric permittivity of granular materials in the 1.7-2.6-GHz band. IEEE Instrum. Meas. 2016, 65, 1051-1059. [CrossRef]

20. Grignon, R.; Afsar, M.N.; Yong, W.; Butt, S. Microwave Broadband Free-Space Complex Dielectric Permittivity Measurements on Low Loss Solids. In Proceedings of the 20th IEEE Instrumentation Technology Conference (Cat. No.03CH37412), Vail, CO, USA, 20-22 May 2003; pp. 865-870. [CrossRef]

21. Lee, I.-G.; Yoon, Y.-J.; Choi, K.-S.; Hong, I.-P. Design of an optical transparent absorber and defect diagnostics analysis based on near-field measurement. Sensors 2021, 21, 3076. [CrossRef]

22. Jonathan, H.; Andrew, J.G.; Claudio, B. Free-space permittivity measurement at terahertz frequencies with a vector network analyzer. IEEE Trans. Terahertz Sci. Technol. 2016, 6, 817-823. [CrossRef]

23. Xiaoning, Y. De-embedding errors due to inaccurate test fixture characterization. IEEE Electromagn. Compat. Mag. 2012, 1, 75-78. [CrossRef]

24. Keysight Technologies. De-embedding and Embedding S-Parameter Networks Using a Vector Network Analyzer; Application Note 1364-1; Keysight Technologies: Santa Rosa, CA, USA, 2016.

25. Srivastava, N.; Hinton, G.; Krizhevsky, A.; Sutskever, I.; Salakhutdinov, R. Dropout: A simple way to prevent neural networks from overfitting. J. Mach. Learn. Res. 2014, 15, 1929-1958. 
26. Nauman, M.; Kim, H.-J.; Song, S.-J.; Kang, S.-S. Investigation of deep neural network with drop out for ultrasonic flaw classification in weldments. J. Mech. Sci. Technol. 2018, 32, 3073-3080. [CrossRef]

27. Hinton, G.E.; Srivastava, N.; Krizhevsky, A.; Sutskever, I.; Salakhutdinov, R.R. Improving neural networks by preventing co-adaptation of feature detectors. arXiv 2012, arXiv:1207.0580.

28. Phil, K. MATLAB Deep Learning: With Machine Learning, Neural Networks and Artificial Intelligence; Apress: Seoul, Korea, 2017; pp. 103-120. ISBN 978-1-4842-2844-9.

29. Nauman, M.; Kim, H.-J.; Song, S.-J.; Kang, S.-S. Convolutional neural network for ultrasonic weldment flaw classification in noisy conditions. Ultrasonics 2019, 94, 74-81. [CrossRef]

30. Chandra, S.A.; Helena, G.R.; Tiago, J.R.; Artur, L.R. SVM Classification of Thickness and Lift-Off Using Transient Eddy Current Oscillation Method. In Proceedings of the 2016 IEEE International Instrumentation and Measurement Technology Conference Proceedings, Taipei, Taiwan, 23-26 May 2016; pp. 1-6. [CrossRef]

31. Iwan, S.; Adam, P.B.; Gary, W. SVM Parameter optimization using grid search and genetic algorithm to improve classification performance. Telkomnika 2016, 14, 1502-1509. [CrossRef] 